\title{
Numerical analysis of the effect of illumination intensity on photoelectric parameters of the silicon solar cell with various metal nanoparticles
}

\author{
J. Gulomov, R. Aliev \\ Department of physics, Andijan state university, Andijan, 119, St. Universitet, 170100, Uzbekistan \\ jasurbekgulomov@yahoo.com, alievuz@yahoo.com
}

PACS 85.60.Bt, 78.20.Bh, 73.61.Ga

DOI 10.17586/2220-8054-2021-12-5-569-574

\begin{abstract}
It is important to study the effect of light intensity on the main photoelectric parameters of silicon solar cell with various metal nanoparticles because the intensity of sunlight is variable. In this paper, the effect of $\mathrm{Cu}, \mathrm{Pt}, \mathrm{Au}, \mathrm{Ag}, \mathrm{Ti}, \mathrm{Al}$, Co nanoparticles on dependence of main photoelectric parameters of silicon solar cell on light intensity has been studied by modeling with Sentaurus TCAD. The intensity coefficient of short circuit current densities of Pt and Ti nanoparticles induced silicon solar cells were found to be $K_{J, P t}=0.0158 \mathrm{~A} / \mathrm{W}$ and $K_{J, T i}=0.0164 \mathrm{~A} / \mathrm{W}$. For simple silicon solar cell, this value was found to be $K_{J}=0.0071 \mathrm{~A} / \mathrm{W}$. Thus, we have observed that was the two-fold greater the intensity coefficient of short circuit current density and output power for the silicon solar cells with Ti and Pt nanoparticles relative to that of a simple silicon solar cell.
\end{abstract}

Keywords: metal nanoparticles, solar cell, nanoplasmonics, modeling, intensity.

Received: 10 July 2021

Revised: 17 August 2021

\section{Introduction}

Solar cells are especially used in the outdoor environment. The changing of seasons has an influence on the operation of solar cells because, luminosity is changed. The influence of light intensity on main photoelectric parameters of silicon solar cells [1] and panels [2] were studied properly. But for nanoparticles induced silicon solar cells (NISSC), it hasn't been thoroughly researched yet. The effect of gold and silver nanoparticles on the optical properties of silicon solar cells was studied experimentally [3] and simulated by using Lumerical TCAD [4]. In our previous papers, the optimal conditions in inserting nanoparticles into silicon solar cells were found. Acceptable size of nanoparticles was $20 \mathrm{~nm}$, while the optimal distance between adjacent nanoparticles was $100 \mathrm{~nm}$. Moreover, nanoparticles should ideally have been inserted into the $n$ region of silicon solar cell [5]. In this paper, the influence of illumination intensity on main photoelectric parameters of NISSCs have been investigated as a logical continuation of our previous research. A modeling method has been used. There are several TCAD programs to model semiconductor devices. The most reliable and best-known programs among them are: Sentaurus TCAD, Lumerical, Silvaco TCAD as well as Comsol Multiphysics. Among their advantages, the obtained results are more reliable and often are similar to experimental results [6-8]. Besides, from prior literature, some information about the comparing experiment results and simulation results, which are obtained by using TCAD programs, are given $[9,10]$. Moreover, all types of semiconductor devices can be modeled by using them.

\section{Method}

To explore NISSCs, simulation methods are mainly used. In this work, models of NISSCs have been created by using Sentaurus TCAD packages.

In Sentaurus TCAD, only four main tools are used to simulate solar cells: Sentaurus Structure Editor, Sentaurus Device, Sentaurus Visual and Sentaurus Workbench. Sentaurus Workbench is environment that assists all interconnected tools to work. From tools, which are added to use in Sentaurus Workbench, is used only by writing codes in tool command language (TCL). For example, the geometrical model of a solar cell is created by writing codes in Sentaurus Structure Editor, which is added to the Sentaurus Workbench environment. This method is more effective, but the Sentaurus Structure Editor has an SDE module to create geometrical model of solar cells by using standard shapes. After creating geometric model in that module, codes can be generated for use in the Sentaurus Structure Editor command file. So, different models must be created for every project. To form geometrical model of various solar cells by creating unique algorithm in TCL is acceptable way. Therefore, in this paper, geometrical model of NISSC has been created by writing codes in TCL. In our previous scientific works, we gave full information about creating geometric models of various solar cells [11]. 
After creating geometrical model of solar cell, physical properties were given, and then calculations were performed by using Sentaurus Device. Physical properties of all materials were uploaded to model as a parameter file. Physical models were added to simulation by writing the code in the Sentaurus Device command file.

The simplest state among the processes, which occur in the semiconductor devices, is the equilibrium state. In this state, the Poisson equation, which is shown in formula (1), must be solved:

$$
\Delta \phi=-\frac{q}{\varepsilon}\left(p-n+N_{D}+N_{A}\right)
$$

where $\varepsilon$ is thepermittivity, $n$ and $p$ are the concentrations of the electrons and holes, $N_{D}$ and $N_{A}$ are the donor and acceptor concentrations, and $q$ is the electron charge.

Electron concentration $\mathrm{n}$ as well as hole concentration $\mathrm{p}$ are calculated by using Fermi distribution that is shown formula (2) and (3):

$$
\begin{aligned}
& n=N_{c} F_{1 / 2}\left(\frac{E_{F, n}-E_{c}}{k T}\right), \\
& p=N_{V} F_{1 / 2}\left(\frac{E_{V}-E_{F, p}}{k T}\right),
\end{aligned}
$$

where: $F_{1 / 2}$ is the Fermi half integral, $E_{c}$ is the conduction band energy, $E_{v}$ is the valence band energy, $E_{F, n}$ is the quasi fermi energy for electrons, $E_{F, p}$ is the quasi fermi energy for holes, $T$ is the absolute temperature, $N_{c}$ is the density of states in conduction band, $N_{v}$ is the density of states in valence band, and $k$ is Boltzmann constant.

Transportation of carriers occurred due to externally applied voltage, illumination or heating. In these phenomena, the equilibrium state is disrupted; due to solar cells being illuminated, carrier transport should also be calculated during simulation in them. The motion of the carriers forms a current. Basically, connection between carrier concentration and current density is expressed by "general continuity equations" which are shown in formula (4) and (5):

$$
\begin{aligned}
& \nabla \cdot \vec{J}_{n}=q R_{n e t, n}+q \frac{\partial n}{\partial t}, \\
& -\nabla \vec{J}_{p}=q R_{n e t, p}+q \frac{\partial p}{\partial t},
\end{aligned}
$$

where: $J_{n}, J_{p}$ are the current densities of electron and holes, $R_{n e t, p}, R_{n e t, n}$ are the net recombination of electron and holes, $t$ - time.

In Sentaurus Device, "Drift-Diffusion", "Thermodynamic", "Hydrodynamic" and "Monte Carlo" models are used to calculate carrier transport. The "Thermodynamic" models, which are given in formulas (6) and (7), have been used to model solar cell, since, generation and recombination rate of carriers is changed when light intensity is changed. Additionally, heat energy or phonon concentration can also be changed. Therefore, heat energy, which is formed due to motion of carriers, also changed. All of these have been taken into account in "Thermodynamic" models, which are given formulas (6) and (7).

$$
\begin{gathered}
\overrightarrow{J_{n}}=-n q \mu_{n}\left(\nabla \Phi_{n}+P_{n} \nabla T\right), \\
\vec{J}_{p}=-p q \mu_{p}\left(\nabla \Phi_{p}+P_{p} \nabla T\right),
\end{gathered}
$$

where: $\mu_{n}, \mu_{p}$ are the mobility of electron and holes, $\Phi_{n}, \Phi_{p}$ are the electron and hole quasi-Fermi potentials, $P_{n}, P_{p}$ are thermoelectric power of electron and holes, $T$ is the absolute temperature.

Formula (8) has been used to calculate carriers transport in metal nanoparticles:

$$
\vec{J}_{M}=-\sigma\left(\nabla \Phi_{M}+P \nabla T\right),
$$

where: $\sigma$ is theconductivity of metal, $\Phi_{m}$ is the Fermi potential in metal, $P$ is the metal thermoelectric power, $J_{M}$ is current density in the metal.

It has been considered that there were ohmic boundary conditions between the metal nanoparticles and silicon interface, due to nanoparticles input into $\mathrm{n}$ region of silicon solar cell. Ohmic boundary conditions were calculated by formula (9):

$$
\begin{aligned}
\vec{J}_{M} \cdot \hat{n} & =\left(\overrightarrow{J_{n}}+\vec{J}_{p}+\vec{J}_{D}\right) \cdot \hat{n}, \\
\phi & =\Phi_{M}-\Phi_{0}, \\
n & =n_{0}, \\
p & =p_{0},
\end{aligned}
$$

where: $J_{D}$ is the diffusion current density, $\hat{n}$ is the normal vector, $\Phi_{0}$ is the equilibrium electrostatic potential (the built-in potential), $\phi$ - electrostatic potential; $n_{0}, p_{0}$ are the electron and hole equilibrium concentrations. 
In this paper, AM1.5G was chosen as a light source. For AM1.5G spectrum [12], one sun equals $633 \mathrm{~W} / \mathrm{m}^{2}$. The intensity of light, which is falling on the solar cell, was varied from 0.1 sun to 1 sun, but the quality of the spectrum wasn't changed, as is shown Fig. 1.

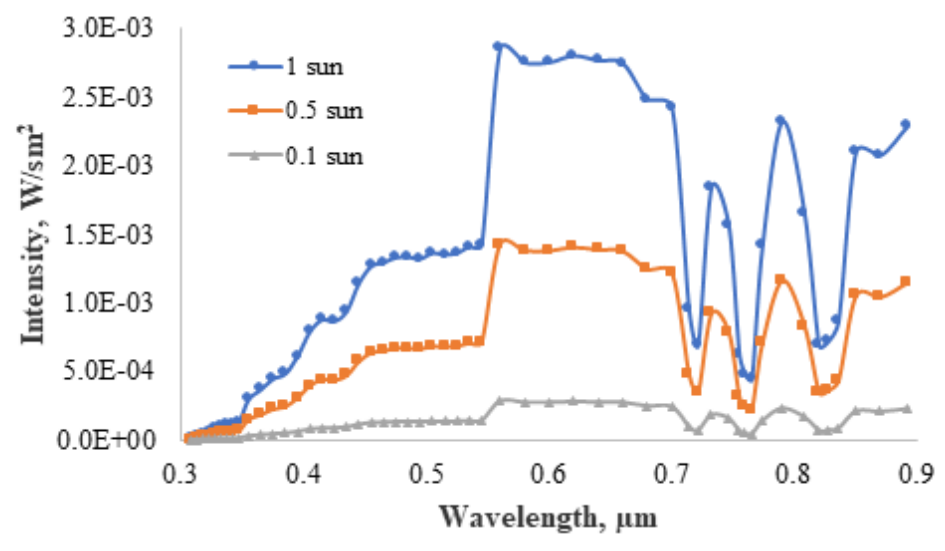

FIG. 1. AM1.5G light spectrum with various intensities

Tables of dependence of complex refractive index of materials on light wavelength were used to calculate the optical properties of silicon solar cells. The total absorption, reflection and transmission factors of solar cells were calculated by using "Transfer matrix method".

Various AM1.5G spectrum files were created. Then, in Sentaurus Workbench, different values for each file were attached. In Sentaurus Device attached values were checked by the conditional operators, then necessary spectrum file was uploaded to model.

\section{Results and discussion}

In our previous research work, the optimal conditions of nanoparticles size, the distance between neighboring nanoparticles and their location were found. By using those conditions, the dependence of main photoelectric parameters of NISSCs on illumination intensity have been studied in this paper. In Fig. 2, the dependence of absorption coefficient of SSC and $\mathrm{Cu}, \mathrm{Au}, \mathrm{Ag}$, Pt NISSCs on light wavelength are given. With light of $0.5 \mu$ m wavelength, resonance event was occurred for all type NISSCs. In studies by Bonaccorso, the resonance of absorption coefficient of gold nanoparticles with interval of radius 10 to $30 \mathrm{~nm}$ occurred with light a wavelength of $0.5 \mu \mathrm{m}$ [13]. These experiments prove the reliability of our simulation results. However, the maximum absorption coefficient was observed in Pt NISSC. In addition, absorption coefficient of Pt NISSC in the infrared field was enhanced. Additionally, in the Brady's experimental work, influence of nanoparticles on the properties silicon solar cell was properly studied [14].

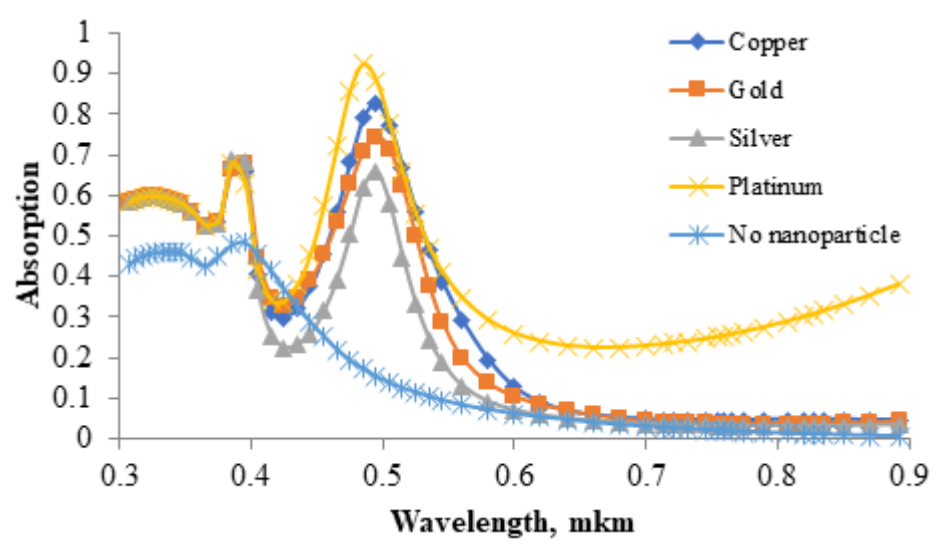

FIG. 2. Dependence of absorption coefficient of SSC and $\mathrm{Cu}, \mathrm{Au}, \mathrm{Ag}, \mathrm{Pt}$ NISSCs on light wavelength

The main parameters of solar cells are determined from I-V characteristics. In Fig. 3, I-V characteristics of SSC and Pt, Ag NISSCs are given. Because, only Pt and Ag nanoparticles had a tangible effect on the I-V characteristics 
of SSC. Au and $\mathrm{Cu}$ nanoparticles had negligible effects. Pt nanoparticle doubled the current density of SSC, due to platinum having a high absorption coefficient. Ag nanoparticle reduced the current density of SSC by 1.2 fold. This phenomenon was observed in Tayyar Dzhaharov's experimental research [15]. The Fano interference can explain this result [16].

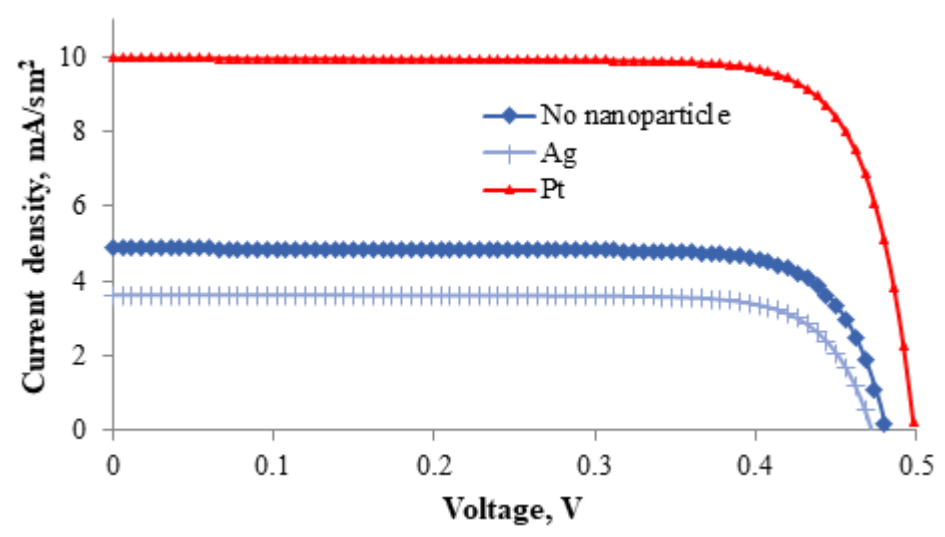

FIG. 3. I-V characteristics of SSC and Ag, Pt NISSCs

Photoelectric parameters change due to varying light intensity. In Fig. 4, the dependence of short circuit current density of SSC and Ag, Pt, Cu NISSCs on the illumination intensity is shown. Light intensity coefficient of short circuit current was found to be $K_{J}=0.0071 \mathrm{~A} / \mathrm{W}$ for SSC, $K_{J, P t}=0.0158 \mathrm{~A} / \mathrm{W}$ for Pt NISSC, $K_{J, A g}=0.000575 \mathrm{~A} / \mathrm{W}$ for Ag NISSC and $K_{J, C u}=0.00831 \mathrm{~A} / \mathrm{W}$ for Cu NISSC. In Chegar's experimental work, the intensity coefficient of short circuit current found was $K_{J}=0.0051 \mathrm{~A} / \mathrm{W}$ for SSC and the dependence of short circuit current on light intensity was linear [17]. It was found to be intensity coefficient of Pt NISSC 2.05 times greater than that of SSC. In the research of Bao, it was found that short circuit current of solar cell, in which Pt nanoparticles were deposited by using one minute electroless deposition, was 2.27 times greater than that of solar cell without Pt nanoparticles [18]. In the Au nanoparticles, the dependence of short circuit current of SSC on light intensity was hardly ever affected. The intensity coefficient of short circuit current of Au NISSC was found to be 1.34 times worse than that of SSC.

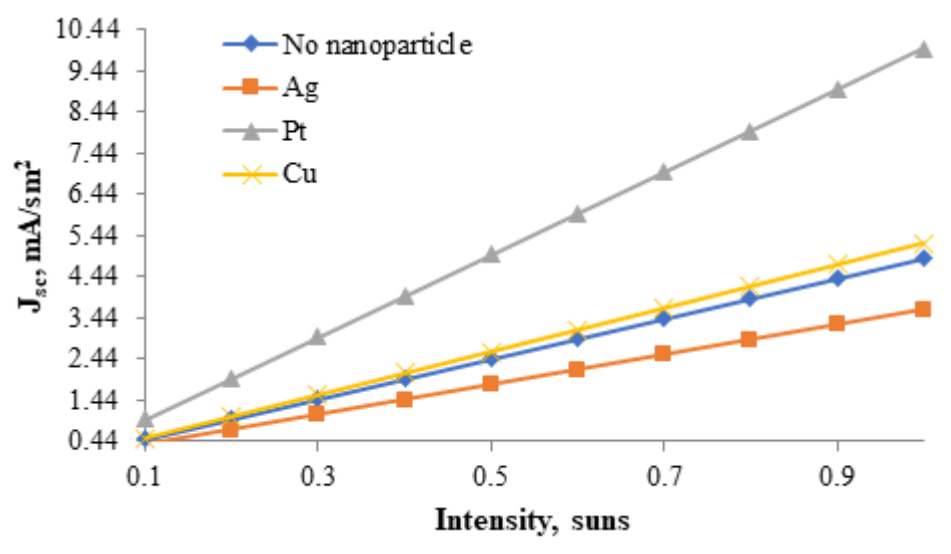

FIG. 4. Dependence of short circuit current density of SSC and NISSC on light intensity

Dependence of the open circuit voltage of SSC on light intensity is known from other research works to be nonlinear [19]. In Fig. 5, dependence of open circuit voltage of SSC and Au, Ag, Pt NISSCs on light intensity are shown. The intensity coefficients of open circuit voltage of SSC and NISSCs were determined to be the same, $K_{U}=1.05 \mathrm{E}-4 \mathrm{Vcm}^{2} / \mathrm{W}$. Thus, the nanoparticles affected the value of open circuit voltage of SSC, but they did not affect the intensity coefficient of open circuit voltage of SSC. In some studies, the dependence of open circuit voltage on light intensity is explained with changing short circuit current density. So, short circuit current linearly depends on light intensity. As shown formula (10), the open circuit voltage has a logarithmic dependence on the short circuit 
current [20]. So, between open circuit voltage and light intensity has logarithmic dependence:

$$
V_{O C}^{\prime}=\frac{n k T}{q} \ln \left(\frac{X I_{S C}}{I_{0}}\right)=\frac{n k T}{q}\left[\ln \left(\frac{I_{S C}}{I_{0}}\right)+\ln X\right]=V_{O C}+\frac{n k T}{q} \ln X,
$$

where: $V_{O C}$ is open circuit voltage in one sun intensity, $V_{O C}^{\prime}$ is the open circuit voltage, $X$ is concentration coefficient, $I_{S C}$ is short circuit current, $I_{0}$ is dark current.

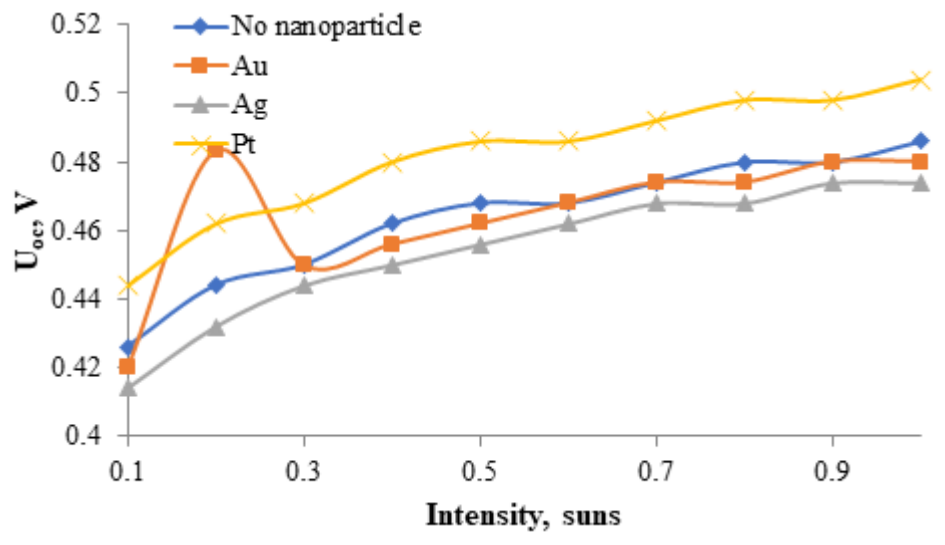

FIG. 5. Dependence of open circuit voltage of SSC and Au, Ag, Pt NISSCs on light intensity

The quality of solar cells is evaluated by their fill factor. In Fig. 6, dependence of fill factor of SSC and Au, Ag, $\mathrm{Pt}, \mathrm{Cu}$ NISSCs on light intensity is shown. The dependence of fill factor on light intensity is not linear. In this paper, it was found that fill factor increased until 0.6 solar intensity, then it decreased. This occurred due to increasing of series resistance in high intensity [21]. These obtained results are similar to those obtained by Khan [22].

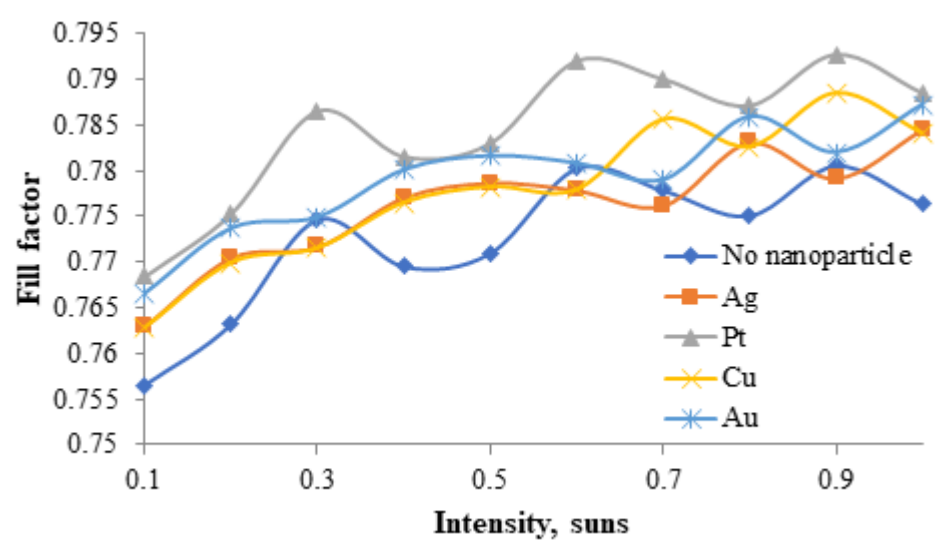

FIG. 6. Dependence of fill factor of SSC and Au, Ag, Pt, Cu NISSCs on light intensity

Various metals can be input into silicon solar cells as nanoparticles. Every metal nanoparticle can exert various effects on silicon solar cell properties. In Table 1, light intensity coefficients of main photoelectric parameters of SSC and NISSCs are given. Nanoparticles have affected all photoelectric parameters except open circuit voltage and voltage on maximum power point.

\section{Conclusion}

The spectral sensitivity and efficiency of solar cells have been enhanced due to considerable research. In this paper, effects of light intensity on photoelectric parameters of various NISSCs have been studied. Thus, it was determined that the effects of $\mathrm{Pt}$, Ti nanoparticles were favorable, the effects of $\mathrm{Ag}$, Co nanoparticles were deleterious, and the effects of $\mathrm{Au}, \mathrm{Cu}$ were negligible. The obtained results show that properties of SSCs can be changed by inputting palpable nanoparticles. It is purposeful to use silicon material, which nanoparticles is input, not only to make solar cells but also other semiconductor materials. For instance, silicon material, to which Pt and Ti nanoparticles can be input, has potential for use in a next-generation pyrometer, which has twice sensitivity. 
TABLE 1. Light intensity coefficients of main photoelectric parameters of SSC and NISSCs

\begin{tabular}{ccccccc}
\hline Nanoparticles & $\begin{array}{c}\mathbf{d U o c} / \mathbf{d W}, \\
\mathbf{V s m}^{2} / \mathbf{m W}\end{array}$ & $\begin{array}{c}\mathbf{d I s c} / \mathbf{s W}, \\
\mathbf{A} / \mathbf{W}\end{array}$ & $\begin{array}{c}\mathbf{d U m p p} / \mathbf{d W}, \\
\mathbf{V s m}^{\mathbf{2}} / \mathbf{m W}\end{array}$ & $\begin{array}{c}\mathbf{d I m p p} / \mathbf{d W}, \\
\mathbf{A} / \mathbf{W}\end{array}$ & $\mathbf{d P m p p} / \mathbf{d W}$ & $\begin{array}{c}\mathbf{d F F} / \mathbf{d W}, \\
\mathbf{s m}\end{array} \mathbf{m W}$ \\
\hline $\mathbf{C}$ & $1.05 \mathrm{E}-02$ & $7.71 \mathrm{E}-01$ & $1.05 \mathrm{E}-02$ & $7.13 \mathrm{E}-01$ & $2.96 \mathrm{E}-01$ & $3.49 \mathrm{E}-03$ \\
$\mathbf{C u}$ & $1.05 \mathrm{E}-02$ & $8.31 \mathrm{E}-01$ & $9.48 \mathrm{E}-03$ & $7.78 \mathrm{E}-01$ & $3.22 \mathrm{E}-01$ & $3.74 \mathrm{E}-03$ \\
$\mathbf{A u}$ & $1.05 \mathrm{E}-02$ & $7.40 \mathrm{E}-01$ & $1.05 \mathrm{E}-02$ & $6.86 \mathrm{E}-01$ & $2.84 \mathrm{E}-01$ & $3.62 \mathrm{E}-03$ \\
$\mathbf{A g}$ & $1.05 \mathrm{E}-02$ & $5.75 \mathrm{E}-01$ & $1.05 \mathrm{E}-02$ & $5.32 \mathrm{E}-01$ & $2.17 \mathrm{E}-01$ & $3.77 \mathrm{E}-03$ \\
$\mathbf{P t}$ & $1.05 \mathrm{E}-02$ & $1.58 \mathrm{E}+00$ & $1.05 \mathrm{E}-02$ & $1.47 \mathrm{E}+00$ & $6.37 \mathrm{E}-01$ & $3.53 \mathrm{E}-03$ \\
$\mathbf{A l}$ & $1.05 \mathrm{E}-02$ & $9.20 \mathrm{E}-01$ & $1.05 \mathrm{E}-02$ & $8.54 \mathrm{E}-01$ & $3.59 \mathrm{E}-01$ & $3.62 \mathrm{E}-03$ \\
$\mathbf{C o}$ & $1.05 \mathrm{E}-02$ & $6.11 \mathrm{E}-01$ & $1.05 \mathrm{E}-02$ & $5.62 \mathrm{E}-01$ & $2.30 \mathrm{E}-01$ & $3.49 \mathrm{E}-03$ \\
$\mathbf{T i}$ & $1.05 \mathrm{E}-02$ & $1.64 \mathrm{E}+00$ & $1.05 \mathrm{E}-02$ & $1.53 \mathrm{E}+00$ & $6.64 \mathrm{E}-01$ & $3.51 \mathrm{E}-03$ \\
\hline
\end{tabular}

\section{References}

[1] Stamenic L., Smiley E., Karim K. Low light conditions modelling for building integrated photovoltaic (BIPV) systems. Solar Energy, 2004, 77 (1), P. 37-45.

[2] Bayhan H., Bayhan M. A simple approach to determine the solar cell diode ideality factor under illumination. Solar Energy, 2011, 85 (5), P. 769-775.

[3] Jeng M.J., Chen Z.Y., et al. Improving Efficiency of Multicrystalline Silicon and CIGS Solar Cells by Incorporating Metal Nanoparticles. Materials, 2015, 8 (10), P. 6761-6771.

[4] Shilpa G.D., Subramanyam T.K., Sreelakshmi K., Uttarakumari. Study and Optimization of Metal Nanoparticles for the Enhanced Efficiency Thin Film Solar Cells. IOP Conference Series: Materials Science and Engineering, 2016, 149, 012074.

[5] Aliev R., Gulomov J., et al. Stimulation of Photoactive Absorption of Sunlight in Thin Layers of Silicon Structures by Metal Nanoparticles. Applied Solar Energy, 2020, 56, P. 364-370.

[6] Ghione G. Looking for Quality in TCAD-Based Papers. IEEE Transactions on Electron Devices, 2019, 66 (8), P. 3252-3253.

[7] Pfäffli P., Wong H.Y., et al. TCAD modeling for reliability. Microelectronics Reliability, 2018, 88-90, P. 1083-1089.

[8] Maiti C.K. Introducing Technology Computer-Aided Design (TCAD): Fundamentals, Simulations, and Applications. Jenny Stanford Publishing, Boca Raton, Florida, USA, 2017, 438 p.

[9] Feng P., Kim J., et al. Contact model based on TCAD-experimental interactive algorithm. Int. Conf. on Simulation of Semiconductor Processes and Devices, SISPAD, 2015, P. 238-241.

[10] Korn J., Rücker H., Heinemann B. Experimental verification of TCAD simulation for high-performance SiGe HBTs. IEEE 17th Topical Meeting on Silicon Monolithic Integrated Circuits in RF Systems, 2017, P. 94-96.

[11] Gulomov J., Aliev R., et al. Studying the Effect of Light Incidence Angle on Photoelectric Parameters of Solar Cells by Simulation. Int. Journal of Renewable Energy Development, 2021, 10 (4), P. 731-736.

[12] Gueymard C.A., Myers D., Emery K. Proposed reference irradiance spectra for solar energy systems testing. Solar Energy, 2002, 73 (6), P. 443-467.

[13] Bonaccorso F., Zerbetto M., Ferrari A.C., Amendola V. Sorting Nanoparticles by Centrifugal Fields in Clean Media. The Journal of Physical Chemistry C, 2013, 117 (25), P. 13217-13229.

[14] Brady B., Wang P.H., Steenhoff V., Brolo A.G. Nanostructuring Solar Cells Using Metallic Nanoparticles. Metal Nanostructures for Photonics, 2019, P. 197-221.

[15] Dzhafarov T.D., Pashaev M.A., et al. Influence of silver nanoparticles on the photovoltaic parameters of silicon solar cells. Advances in Nano Research, 2015, 3 (3), P. 133-141.

[16] Giannini V., Francescato Y., et al. Fano resonances in nanoscale plasmonic systems: a parameter-free modeling approach. Nano Lett., 2011, 11 (7), P. 2835-2840.

[17] Chegaar M., Hamzaoui A., et al. Effect of Illumination Intensity on Solar Cells Parameters. Energy Procedia, 2013, 36, P. 722-729.

[18] Bao X.Q., Liu L.F. Platinum nanoparticle interlayer promoted improvement in photovoltaic performance of silicon/PEDOT: PSS hybrid solar cells. Materials Chemistry and Physics, 2015, 149-150, P. 309-316.

[19] Chegaar M., Hamzaoui A., et al. Effect of illumination intensity on solar cells parameters. Energy Procedia, 2013, 36, P. $722-729$.

[20] Bunea G., Wilson K., et al. Low Light Performance of Mono-Crystalline Silicon Solar Cells. IEEE 4th World Conference on Photovoltaic Energy Conversion, 2006, P. 1312-1314.

[21] Saloux E., Teyssedou A., Sorin M. Explicit model of photovoltaic panels to determine voltages and currents at the maximum power point. Solar Energy, 2011, 85 (5), P. 713-722.

[22] Khan F., Singh S.N., Husain M. Effect of illumination intensity on cell parameters of a silicon solar cell. Solar Energy Materials and Solar Cells, 2010, 94 (9), P. 1473-1476. 